\title{
Galloway-Mowat syndrome
}

INSERM

\section{Source}

INSERM. (1999). Orphanet: an online rare disease and orphan drug data base. Galloway-

Mowat syndrome. ORPHA:2065

Galloway syndrome is characterized by the association of nephrotic syndrome and central nervous system anomalies. 